\title{
Comparison of diagnostic accuracy and interpretation times for a standard and an advanced 3D visualisation technique in $\mathrm{CT}$ colonography
}

\author{
Thomas Mang • Frank T. Kolligs • Claus Schaefer • \\ Maxmilian F. Reiser • Anno Graser
}

Received: 11 April 2010 /Revised: 6 August 2010 / Accepted: 7 August 2010 / Published online: 3 October 2010

(C) European Society of Radiology 2010

\begin{abstract}
Objective To compare the diagnostic accuracy of a standard bi-directional, three-dimensional (3D) CT colonography (CTC) fly-through (standard view, SV) with a unidirectional, 3D unfolding technique (panoramic view, PV).

Methods 150 consecutive endoscopically-validated CTC patient datasets were retrospectively reviewed twice by two expert radiologists: first, with bidirectional SV, second, after 6-15 months, with unidirectional PV. Per-polyp sensitivities, percentage of visualised colonic mucosa, and reading times were calculated for both $3 \mathrm{D}$ visualisations. Results were tested for statistical significance by equivalence analysis for paired proportions and Student's paired $t$-test.

Results In 81 patients, 236 polyps (101 adenomas, 135 nonadenomas) were detected. Sensitivities for polyps $\leq 5 \mathrm{~mm}$, 6-9 $\mathrm{mm}$ and $\geq 10 \mathrm{~mm}$ were $60.1 \%$ (113/188), 92.9\% (26/28) and $95.0 \%(19 / 20)$ with bidirectional SV, and $60.6 \%$ (114/ 188), $96.4 \%$ (27/28) and $95.0 \%$ (19/20) with unidirectional PV. Overall sensitivity for adenomas was $86.1 \%$ and $84.2 \%$ for SV and PV. Both methods provided equivalent polyp
\end{abstract}

T. Mang $(\bowtie)$

Department of Radiology, Medical University of Vienna,

Waehringer Guertel, 18-20,

1090 Vienna, Austria

e-mail: thomas.mang@meduniwien.ac.at

M. F. Reiser · A. Graser

Department of Clinical Radiology, University of Munich,

Klinikum Grosshadern, Marchioninistrasse 15,

81377 Munich, Germany

F. T. Kolligs $\cdot$ C. Schaefer

Department of Medicine II, University of Munich,

Klinikum Grosshadern, Marchioninistrasse 15,

81377 Munich, Germany detection, with an equivalence limit set at 5\%. PV and SV visualised $98.9 \pm 1.1 \%(97.0-99.9 \%)$ and $96.2 \pm 2.3 \%$ $(91.4-98.8 \%)$ of the colonic mucosa $(p>0.05)$. Mean interpretation time decreased from $14.6 \pm 2.5(9.2-22.8)$ minutes with SV to $7.5 \pm 3.2(5.0-14.4)$ using PV $(p<0.0001)$.

Conclusion 3D CTC interpretation using unidirectional PV is equally as accurate, but significantly faster than an interpretation based on bidirectional SV.

Keywords CT colonography - Virtual colonoscopy · Colorectal polyps $\cdot$ Panoramic view $\cdot$ Three-dimensional evaluation

\section{Introduction}

Computed tomographic colonography (CTC) is an elaborate technique for imaging the large bowel based on thinsection helical computed tomography (CT) of the cleansed and air-distended colon. Generally, a combination of different types of three-dimensional (3D) endoluminal visualisations (virtual endoscopic view) and twodimensional (2D) multiplanar reformatted (MPR) images are used for interpretation [1]. Either 2D or 3D images can be used for the primary search process, with the other technique used for problem solving.

According to recent trials, primary $2 \mathrm{D}$ evaluation is equally as accurate as primary $3 \mathrm{D}$, but potentially less timeconsuming $[2,3]$. When performing a primary $3 \mathrm{D}$ read, $2 \mathrm{D}$ axial views and MPRs are necessary to characterise findings and evaluate underdistended or collapsed segments. CTC 3D standard views, commonly used for primary $3 \mathrm{D}$ evaluations, are intended to simulate a true 
optical colonoscopic examination in terms of the position of the virtual endoscope, its viewing angle, and intraluminal navigation. However, optical colonoscopy as such is not a perfect examination, because of limited surface visualization, resulting in unseen areas, or so-called "blind spots." When using a standard 3D endoluminal display at CTC, data evaluation is hampered by the same limitations, and must, therefore, be performed in both an antegrade and retrograde fashion in order to see lesions hidden behind haustral folds. This is time-consuming [4].

Recently, several new 3D projections have been created to overcome this limitation by increasing surface visibility [5-8]. The so-called "panoramic view" (PV) tool (Fig. 1) is a $3 \mathrm{D}$ virtual endoscopic projection that renders five faces of a cubic viewing space into one view plane in a continuous fashion [9]. Thus, both sides of the haustral folds are displayed in one single fly-through, potentially allowing for unidirectional evaluation which may be more time-efficient.

The purpose of this study was to evaluate whether a unidirectional 3D panoramic view (PV) evaluation of CTC datasets could display the entire colonic wall, and to assess whether this technique would be equally accurate and faster for detection of colorectal polyps, compared to bidirectional 3D standard view (SV).

\section{Materials and methods}

Patient population

Based on recent studies focusing on reading time and visualisation techniques $[6,7]$ we hypothesised that both interpretation techniques would be equally sensitive in the detection of clinically significant polyps. In order to formally test the equivalence of both visualisations, an equivalence analysis for paired proportions was used [10].The equivalence limit difference (delta value) was set at $5 \%$. We assumed that $8 \%$ of ratings would be discordant. To gain a power of $80 \%$, a minimum number of 198 polyps was required.

Therefore we included 150 consecutive asymptomatic patients of average risk who underwent $\mathrm{CT}$ colonography (CTC) and subsequent video optical colonoscopy (OC) between August 2005 and January 2006 at our institution as part of a larger prospective screening trial [11]. The total number of polyps of all sizes in this cohort of patients was 236. We did not select patients based upon the quality of distension, bowel preparation, or overall image noise criteria. This study was conducted under institutional review board approval and written informed patient consent had been obtained. The study population consisted of 71 male and 79 female subjects with a mean age of $61.7 \pm$ 7.2 years (range, 50-79 years). For the retrospective evaluation, patient CTC datasets were anonymized by encrypting patient-related information.

\section{Patient preparation}

Bowel preparation was described previously [11] and was based on a standard "wet prep" regimen, including four litres of polyethylene glycol solution (PEG) (KleanPrep, Norgine Pharmaceuticals, Marburg, Germany) and a commercially available combination of four tablets $(5 \mathrm{mg}$ each, for a total of $20 \mathrm{mg}$ ) of bisacodyl and $30 \mathrm{ml}$ of sodium phosphate (Prepacol, GuerbetPharma, Sulzbach, Germany). There were $50 \mathrm{ml}$ of iodinated contrast agent iopamidol (Solutrast 300, BraccoAltanaPharma, Milan, Italy) added to the last litre of PEG in order to tag residual fluid.

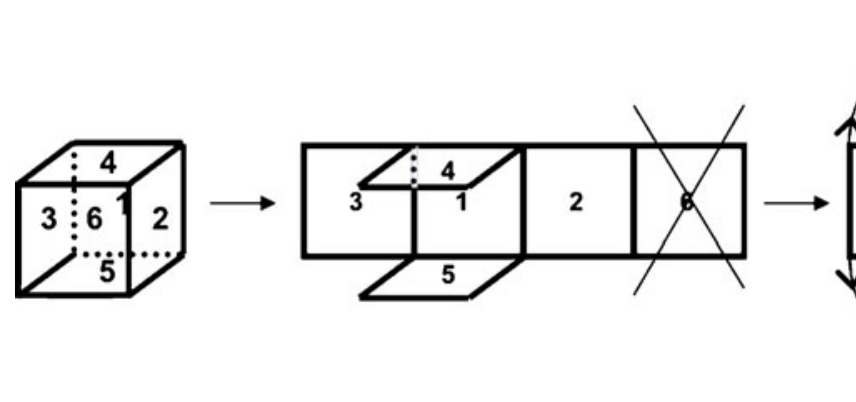

\section{Cube}

Fig. $13 \mathrm{D}$ panoramic projection of the unfolded cube onto a squared display: The panoramic view renders the five faces of a cubic view in a continuous fashion. The virtual camera is placed into a circular space and projects the images in a single plane, using a $90^{\circ}$ viewing angle.
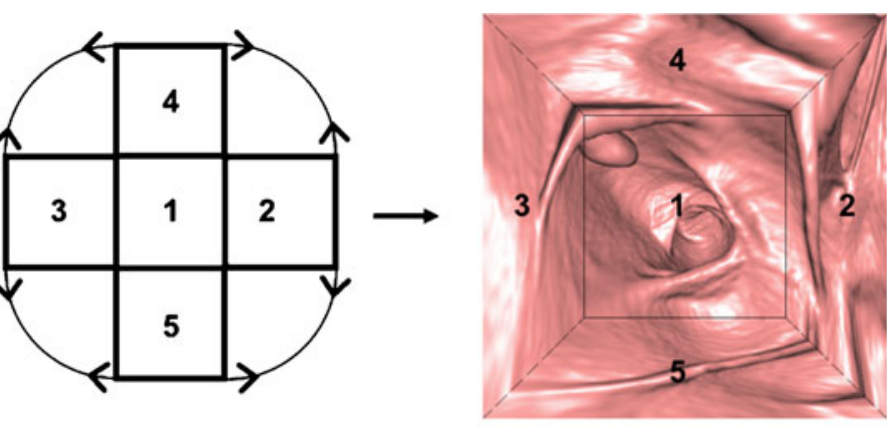

\section{Warping}

The front view is mapped onto a rectangular window in the centre of the plane, and the back projection is removed while the other four images are projected onto a trapezium-shaped window 


\section{CT colonography}

Computed tomographic colonography was performed on a 64-channel multidetector row system (Siemens Somatom Sensation 64, Siemens Medical Solutions, Forchheim, Germany) at a collimation of $0.6 \mathrm{~mm}$ for high-resolution imaging. Images were reconstructed using a standard soft tissue kernel at a slice thickness of $0.75 \mathrm{~mm}$ and a 0.5 $\mathrm{mm}$ reconstruction increment. Tube voltage was $120 \mathrm{kVp}$, and tube current-time product reference values were $70 \mathrm{mAs}$ in the supine and $30 \mathrm{mAs}$ in the prone position. An online dose modulation technique (Care Dose 4D, Siemens Medical Solutions) was used to automatically adapt the tube current to patient anatomy [12]. No intravenous contrast agent was administered. Twenty mg of n-butyl scopolamine (Buscopan, Boeringer Ingelheim Pharmaceuticals, Ingelheim, Germany) were administered intravenously for bowel relaxation immediately before the initiation of bowel distension.

Patients were positioned on the imaging table in the right decubitus position and bowel distension was achieved after placement of a rectal tube by automated $\mathrm{CO}_{2}$ insufflation using a commercially available insufflator (Protocol, Bracco Diagnostics, Princeton, NJ, USA). The adequacy of colonic distension was determined by a radiologist on the CT localiser image of the abdomen. Subsequently, the first set of images was obtained in a 7-9 s breath-hold with the patient in the supine position. After repositioning, the prone dataset was obtained.

Optical colonoscopy and matching of findings

Immediately after CTC, optical colonoscopy (OC) was performed by one of six experienced gastroenterologists using video endoscopy (CF-Q 160 series, Olympus Medical Systems, Hamburg, Germany). Lesions were measured by comparison of their size with an open-biopsy forceps. All polyps were resected or biopsied and retrieved at colonoscopy and sent to histopathology for analysis. For matching of OC and CTC findings, segmental unblinding was used as described elsewhere $[11,13]$. The unblinded OC results were used as the standard of reference.

All findings were documented on a standardised report form. For each of six colonic segments (caecum, ascending colon, transverse colon, descending colon, sigmoid colon and rectum) the absence or presence of polyps was determined and lesion sizes were coded as diminutive $(\leq 5 \mathrm{~mm})$, small (6-9 mm), or large ( $\geq 10 \mathrm{~mm})[1,14]$. In the endoscopy suite, the report form containing the CTC results was provided to one of the endoscopy nurses who revealed the results to the endoscopist after withdrawal of the endoscope from each colonic segment. First- and second-look detections at OC were documented separately. A lesion was rated a truepositive detection if OC and CTC detected a polyp in the same or an adjacent segment of the colon, and if the measured size of the lesion was within the same size category, or if there was a deviation of no more than one size category.

\section{Readers}

All CTC patient datasets were interpreted independently by one of two readers who were blinded to the standard of reference. The readers were abdominal radiologists and experts in CTC who had interpreted a minimum of 500 examinations before this study.

Both readers were familiar with both the reading software programs, and had experience in the evaluation of a minimum of $120 \mathrm{CTC}$ datasets with the 3D panoramic view software. None of the readers was involved in the selection or matching of the study cases. The readers were not aware of the number or size ranges of polyps contained in the study cohort, or the number of positive or negative cases.

\section{Computer workstation and display techniques}

Image processing and interpretation were performed on a commercially available CTC system (Siemens MMWP 2008), into which the PV as well as the SV algorithm had been integrated. The CTC system allows manual, mousedriven, virtual fly-through of the surface-rendered 3D images of both $3 \mathrm{D}$ visualisations with automated real-time centreline path calculation at a user-defined speed. Individual interactive navigation of the virtual endoscope around and beyond the central path is possible. Axial and multiplanar 2D image displays were available.

The panoramic view is a real-time, interactive virtual endoscopic visualisation technique, designed to obtain a contiguous projection, a large field of view and limited distortion. It is a variation of the unfolded cubic view, which renders five faces of a cubic view in the plane in a continuous fashion. Therefore, the front view is mapped into a square while the other four faces are mapped around it into a square using a 90-degree viewing angle to minimise distortions [9] (Fig. 1). This projection is designed to guarantee a smooth transition between the five faces and to remove the discontinuities found in standard cubic projections. The distortion is strongest closer to the periphery of the endoluminal 3D display and weakens towards the centre, displaying the frontal view with no deformation. This imaging technique potentially increases overall colonic wall visualisation, especially of blind spots between or behind haustral folds, and is, therefore, designed to allow for a timeeffective, single retrograde evaluation. 
The standard view simulates a true conventional colonoscopic examination, in terms of navigation, with a standard 110-degree viewing angle.

\section{CTC interpretation}

\section{Assessment of sensitivity}

Each CTC patient dataset was evaluated twice in two separate reading sessions, and the readers were blinded to the results of the colonoscopy. The reader used 3D views for the primary search process at his own preferred evaluation speed. Axial and multiplanar 2D image displays were used for correlation and characterisation of any abnormality detected in 3D.

In the first reading session, the readers evaluated each CTC dataset with standard, bidirectional, 3D virtual endoscopy (standard view, SV), from the rectum to the caecum and back.

In the second part of the evaluation, CTC patient datasets were evaluated with a 3D panoramic view (PV) in a retrograde direction from the rectum to the caecum.

Evaluation was performed at both reading sessions, with the studies displayed in a random order, first in the supine and then in the prone position. To avoid a learning bias by the readers, there was a time interval of 6-15 months between the two reading sessions.

All suspected polyps were recorded by placing a digital marker that also saved the coordinates of the lesion. A polyp was defined as a sessile or stalked, round, oval, or lobulated intraluminal non-mobile filling defect with homogeneous soft tissue attenuation [15]. Findings marked by the reader in each evaluation were compared with the standard of reference to be classified as either true-positive or false-positive findings.

False-positive diagnoses of the reader were assessed for the different $3 \mathrm{D}$ viewing techniques and analysed with regard to the number and reason for the false-positive diagnoses.

\section{Assessment of surface display}

In addition, the area of colonic mucosa, visualised on a 3D fly-through from rectum to caecum, was recorded for unidirectional retrograde $\mathrm{PV}$, unidirectional retrograde SV and for the bidirectional SV. This was achieved using a research software tool that had been integrated into the workstation ("Unseen Areas" tool, Siemens MMWP version VA 30) that automatically highlights unseen areas of the colonic mucosa when activated. The tool also calculates the percentage of unseen areas in relation to the total endoluminal mucosal surface area during real-time data evaluation by the reader. The seen and unseen colonic surface can be depicted easily on the workstation after the review by painting with a distinct colour on the endoluminal and on the global view.

Assessment of reading time

The interpretation time used for complete colonic evaluation of each case was recorded for both bidirectional SV and unidirectional $\mathrm{PV}$, in order to allow for comparison of the time effectiveness of the two different $3 \mathrm{D}$ approaches.

\section{Statistical analysis}

Descriptive statistics including calculation of sensitivities according to polyp size (diminutive, small and large) and polyp histology (adenomatous versus non-adenomatous), with bidirectional SV and unidirectional PV, were performed on a per-polyp basis. Calculation of specificity for both 3D visualisations was based on colonic segments. The significance of difference was tested using the McNemar Test.

In order to formally test the equivalence of both visualisations, an equivalence analysis for paired proportions was used [10]. The null hypothesis for this type of analysis is that the tests are not equivalent; the alternative hypothesis is that they are equivalent.

The equivalence limit difference (delta value) was set at $5 \%$. Both reading strategies would be considered equivalent if the confidence interval for the difference in sensitivity fell entirely within the zone of equivalence (interval \pm 0.05 or $\pm 5 \%$ ).

Differences in the percentage of visualised colonic mucosa were tested for statistical significance using the paired Student's $t$-test, which was also employed to assess the significance of differences in reading time for the SV versus the $\mathrm{PV}$ reading paradigm.

\section{Results}

\section{Standard of reference}

Tables 1 and 2 summarise the distribution of polyps according to size and location. A total of 900 colonic segments were analysed in 150 patients. All 236 endoscopically proven polyps, $101(43 \%)$ adenomas, and 135 $(57 \%)$ non-adenomas, could be identified in retrospect on the CTC images in at least one of the two series in 81 patients. The smallest size lesions that were recorded measured $2 \mathrm{~mm}$. Of the 150 patients, 69 had normal results at $\mathrm{CTC}$ and $\mathrm{OC}$. 
Table 1 Histology and size of lesions detected on the reference standard

\begin{tabular}{|c|c|c|c|c|c|}
\hline & & Total & $0-5 \mathrm{~mm}$ & $6-9 \mathrm{~mm}$ & $\geq 10 \mathrm{~mm}$ \\
\hline \multirow[t]{3}{*}{ Polyps - all } & total number & 236 & 188 & 28 & 20 \\
\hline & adenomas & 101 & 56 & 25 & 20 \\
\hline & non-adenomas & 135 & 132 & 3 & 0 \\
\hline \multicolumn{6}{|c|}{ Polyps - adenomas } \\
\hline & total number & 101 & 56 & 25 & 20 \\
\hline & tubular & & 43 & 18 & 10 \\
\hline & tubulovillous & & 8 & 3 & 4 \\
\hline & villous & & 4 & 4 & 5 \\
\hline & carcinoma & & 0 & 0 & 1 \\
\hline & serrated & & 1 & 0 & 0 \\
\hline & high-grade dysplasia & 1 & 1 & 2 & \\
\hline \multicolumn{6}{|c|}{ Polyps - non-adenomas } \\
\hline & total number & 135 & 132 & 3 & 0 \\
\hline & hyperplastic & 122 & 120 & 2 & 0 \\
\hline & inflammatory & 12 & 11 & 1 & 0 \\
\hline & other & 1 & 1 & 0 & 0 \\
\hline
\end{tabular}

\section{Surface visualisation}

The mean surface coverage after unidirectional retrograde fly-through with PV was 98.9 $\pm 1.1 \%$ (range, 97.0-99.9\%) of the colonic mucosa, whereas the unidirectional SV covered only $79.4 \pm 4.0 \%$ (range, 67.5-85.0\%) $(p<0.0001)$. Conversely, the mucosal coverage with a bidirectional standard view was $96.2 \pm 2.3 \%$ (91.4-98.8\%) (Table 3, Fig. 2). The difference in coverage between unidirectional retrograde PV and bidirectional SV was not statistically significant $(p>0.05)$.

\section{Reader performance}

The results regarding lesion detection are shown in Table 4. For standard bidirectional SV, the overall sensitivity of the readers for all polyps was $66.9 \%(158 /$ 236): $86.1 \%(87 / 101)$ for adenomas and 52.6\% (71/135) for non-adenomas. With regard to lesion size, the

Table 2 Histology of lesions detected on the reference standard according to location

\begin{tabular}{lccc}
\hline & Adenomas & Non-adenomatous polyps & Total \\
\hline Cecum & 10 & 12 & 22 \\
Ascending & 20 & 12 & 32 \\
Transverse & 12 & 17 & 29 \\
Descending & 13 & 12 & 25 \\
Sigmoid & 41 & 42 & 83 \\
Rectum & 5 & 40 & 45 \\
All segments & 101 & 135 & 236 \\
\hline
\end{tabular}

sensitivity was $95.0 \%$ (19/20) for large polyps $\geq 10 \mathrm{~mm}$, 92.9\% (26/28) for small polyps 6-9 $\mathrm{mm}$ and 60.1\% (113/ 118 ) for diminutive polyps. Equivalence analysis for detection of colonic polyps showed equivalence of the two visualisation methods with a $5 \%$ limit. For the unidirectional retrograde $\mathrm{PV}$, the overall sensitivity of the readers for all polyps was $68.2 \%(161 / 236), 84.2 \%(85 / 101)$ for adenomas and $56.3 \%(76 / 135)$ for non-adenomas (Figs. 3, 4). All standard deviations of sensitivities lie well within the zone of equivalence.

Focusing only on adenomatous polyps, the sensitivity was 95\% (19/20) for large and 96\% (24/25) for small lesions for both $3 \mathrm{D}$ visualizations; for diminutive adenomas, it was $73.2 \%(41 / 56)$ with unidirectional PV, and $78.6 \%(44 / 56)$ with bidirectional SV.

False-positive findings and specificity

The readers made a total of 20 false-positive diagnoses ( $\leq 5 \mathrm{~mm}, n=16 ; 6-9 \mathrm{~mm}, n=4$ ) with bidirectional $\mathrm{SV}$ compared to 28 ( $\leq 5 \mathrm{~mm}, n=23 ; 6-9 \mathrm{~mm}, n=5)$ using the unidirectional retrograde PV. Of the $6-9 \mathrm{~mm}$ findings not confirmed by $\mathrm{OC}, 3 / 5$ on $\mathrm{PV}$ and $2 / 4$ on $\mathrm{SV}$ were attributed to nontagged stool. The remaining two falsepositives were caused by an asymmetrical thickened fold that was misinterpreted as a flat lesion, and by an inverted appendiceal stump that was misinterpreted as a sessile $9 \mathrm{~mm}$ polyp by both visualizations. Diminutive findings $\leq 5 \mathrm{~mm}$ not confirmed by $\mathrm{OC}$, were likely attributable to untagged fecal material in both visualization techniques.

The specificity, calculated on a segmental base, was $97.8 \%$ (880/900 segments) with bidirectional standard SV 
Table 3 Percentage of the colonic surface display for the retrograde panoramic view, and for the bidirectional standard view

\begin{tabular}{lcccc}
\hline Surface display $(\%)$ & & & \\
\hline & Average & Standard deviation & Minimum & Maximum \\
\hline Standard View unidirectional & 79.4 & 4.0 & 67.5 & 85.0 \\
Standard View bidirectional & 96.2 & 2.3 & 91.4 & 98.8 \\
Panoramic View retrograde & 98.9 & 1.1 & 97.0 & 99.9
\end{tabular}

and $96.9 \%(872 / 900$ segments $)$ for the unidirectional retrograde $\mathrm{PV}(p=0.118)$ (Table 4$)$.

\section{Evaluation of interpretation times}

With bidirectional SV, the interpretation time of a complete patient examination ranged from 9.2 to 22.8 (mean, $14.6 \pm$ 3.5). PV evaluation reduced the reading time significantly compared with the SV evaluations. The time for evaluation of a patient dataset for the readers ranged from 5.0 to 14.4 (mean, 7.5 \pm 3.2 ) $\min , p<0.0001$.

\section{Discussion}

There is consensus among experts that a combined 2D and $3 \mathrm{D}$ review should be standard in CTC image interpretation [1]. Although initial reports indicated that 3D might improve sensitivity $[13,16]$, recent results show no significant differences in diagnostic accuracy between primary 3D and primary 2D strategies for the detection of large adenomas, but decreased reading times by about 6 min when a primary 2D approach is used [2]. However, a primary $3 \mathrm{D}$ review of CTC may have some inherent differences compared to a primary $2 \mathrm{D}$ review. It is known that both the conspicuity of polyps and the duration of visualisation are increased by using a 3D endoluminal flythrough, potentially facilitating the initial search process [13]. Because of the limited viewing angle of the virtual endoscope, primary $3 \mathrm{D}$ evaluation requires a bidirectional fly-through to be performed in a retrograde and antegrade fashion for the perception of lesions behind haustral folds which is time-consuming. Recently reported reading times for $3 \mathrm{D}$ standard views range from 10 to $39 \mathrm{~min}[4,17,18]$.

With the growing use of CT colonography, accurate and time-efficient interpretation strategies are needed to accommodate the expected increase in the number of examinations performed with this technique [19]. Therefore, several new 3D projections have been created to enable a single unidirectional evaluation strategy by increasing surface visibility per time unit. These methods aim to provide simultaneous viewing of a greater surface area of the colonic lumen than is displayed by typical 3D endoscopic views. One simple approach is to increase the viewing angle to widen the endoluminal field of view [20-22].

Advanced developments include virtual dissection, filet views, band views, as well as unfolded cube projections and the panoramic view $[6,7,9,19,23]$. Initial results from some advanced 3D displays, tested in a smaller series of patients, showed a reduced reading time without a
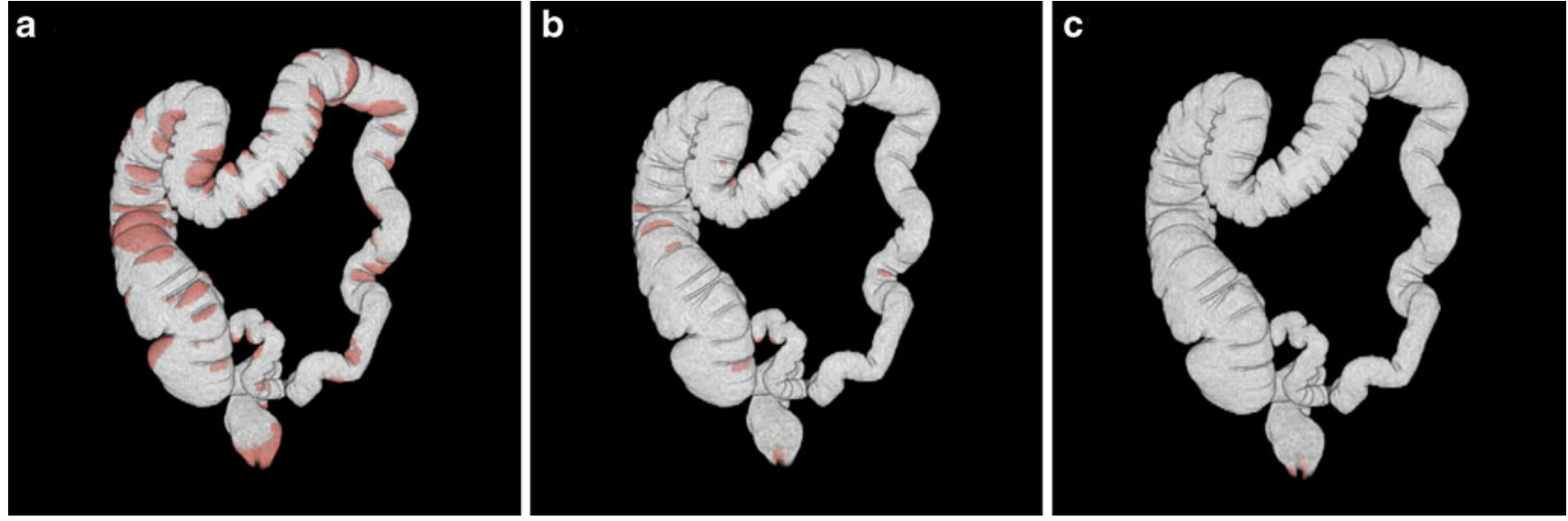

Fig. 2 a-c Surface-rendered 3D global views of the colon. Red areas demonstrate blind spots not displayed by the imaging technique. a Unidirectional retrograde standard virtual endoscopy shows multiple blind spots located behind haustral folds. b Bidirectional retro- and antegrade standard views as well as c a unidirectional retrograde panoramic view significantly reduce the number and size of blind spots 
significant difference in diagnostic accuracy compared with conventional 3D or 2D views $[6,7,24,25]$. To the best of our knowledge, this is the most comprehensive study to evaluate the $3 \mathrm{D}$ panoramic view for quantification of colonic surface display and for the feasibility of polyp detection in an asymptomatic cohort.

Our study indicates that unidirectional PV interpretation results in a significantly faster 3D CTC evaluation. The mean reading time in our cohort with SV virtual endoscopy for the evaluation of a patient dataset consisting of prone and supine images (mean $14.6 \mathrm{~min}$ ) was within the range of results published in the literature [17, 26, 27]. Data evaluation with PV in a single direction reduces the mean reading time by almost $50 \%$ ( $7.5 \mathrm{~min}$ versus $14.6 \mathrm{~min}$ ) compared with a bidirectional SV approach. We included only interpretation time for evaluation of the colon with the $3 \mathrm{D}+2 \mathrm{D}$ approaches described. The additional times for case uploading, extracolonic evaluation and data reporting were not taken into account for both visualisations.

Obviously, a unidirectional evaluation will be more timeefficient than a bidirectional evaluation. However, it is known from 3D standard views that an evaluation in one direction will also result in a significant decrease in sensitivity [4].

The 3D panoramic view also significantly increases surface coverage by rendering the five faces of a cubic view in a continuous fashion (see Fig. 1).

Compared with conventional 3D techniques, the panoramic view displays both sides of haustral folds in a single fly-through for a time-effective single retrograde evaluation. Thus, $98.9 \pm 1.1 \%$ of the colonic surface is displayed, which is significantly more than in a unidirectional SV evaluation $(79.4 \pm 4.0 \%)$, but in the same range as a bidirectional SV evaluation (96.2 $\pm 2.3 \%)$. While PV tends to visualise a very large amount of the mucosa on one flythrough, there might be limitations around sharp turns of the colon, as in a tortuous sigmoid or at the flexures, that can lead to lesions being missed. Whereas unidirectional SV mainly missed areas behind or between haustral folds, bidirectional SV missed only blind spots between narrow standing haustral folds and in some of the inner areas of colonic flexures (see Fig. 2).

The results of the unidirectional and bidirectional surface coverage with the evaluated 3D standard view are within the range of other 3D software solutions in earlier studies. The covered surface percentage was reported, with different software programs, as being between 73 and $76.7 \%$ for unidirectional and 93.8 and $94.1 \%$ for bidirectional SV evaluation $[6,8]$.

With regard to polyp detection, we found equivalence with regard to sensitivity of the two techniques for both adenomas and for non-adenomatous polyps. The unidirectional PV interpretation revealed slightly higher overall per 
Fig. 3 a-d Pedunculated, 8-mm polyp located behind a haustral fold in a 64-year-old asymptomatic male patient. a The polyp is not visible with a retrograde standard view. b Antegrade standard view as well as $\mathbf{c}$ unidirectional retrograde panoramic view display the lesion behind the fold (arrows). Note that the polyp is only displayed on the display periphery. d Transverse 2D image shows the soft tissue attenuation of the polyp (arrow)
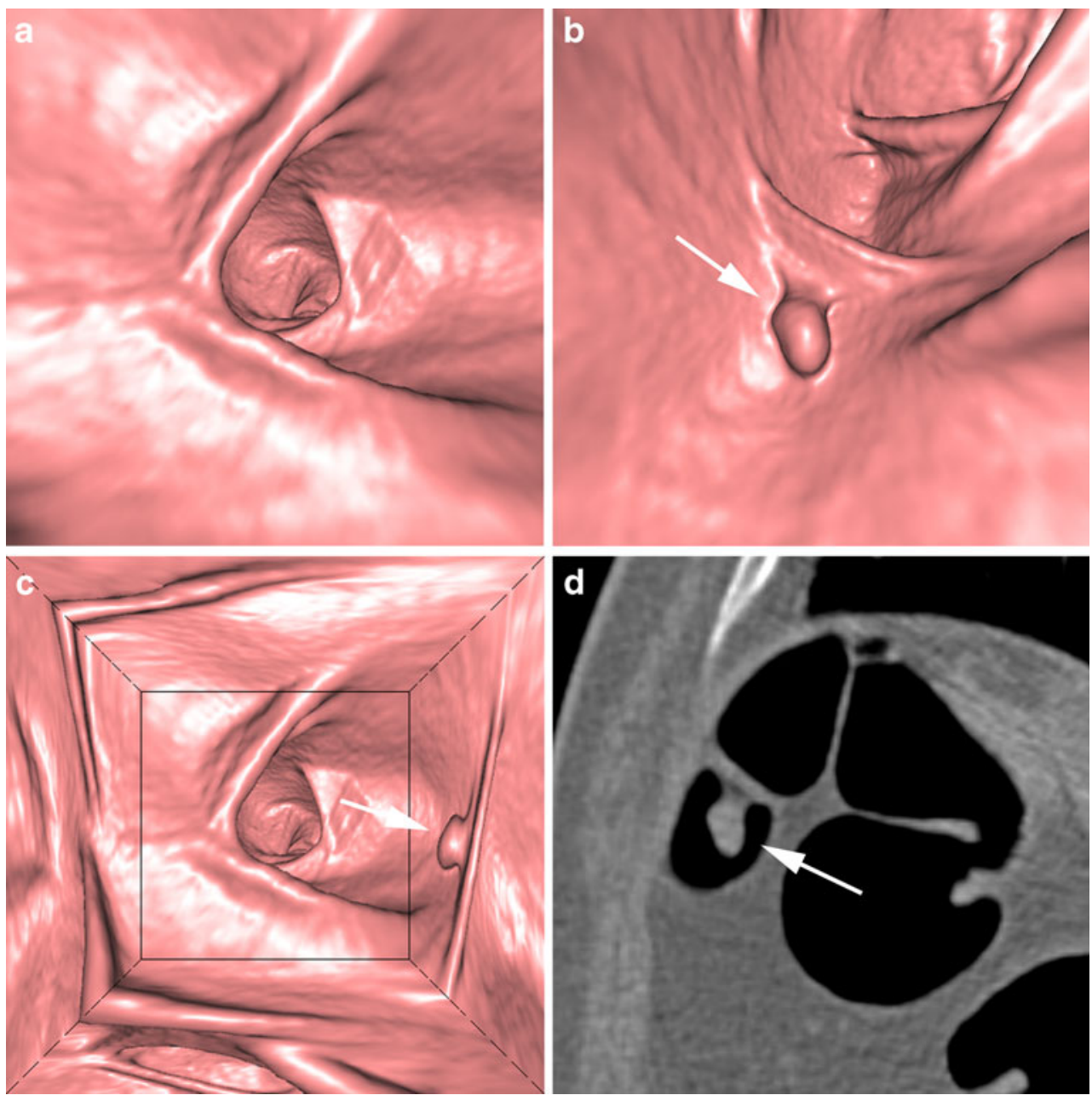

polyp sensitivities compared with bidirectional 3D SV evaluation (68.2\% versus $66.9 \%)$.

Focusing only on clinically relevant adenomas $\geq 6 \mathrm{~mm}$, the sensitivity was equal for both techniques at $96 \%$ (43/45). The sensitivity for diminutive adenomas $<6 \mathrm{~mm}$ was slightly lower with $\mathrm{PV}$ than with $\mathrm{SV}$, but not statistically significantly, $78.6 \%(44 / 56)$ versus $73.2 \%$ (41/ 56). The higher sensitivity for adenomas than for nonadenomatous polyps of the two techniques, SV and PV, might be related to the fact that non-adenomatous lesions in our population were mainly diminutive $(89.4 \%)$ and small (1.6\%), whereas adenomas counted for $94 \%$ of lesions $\geq 6 \mathrm{~mm}$. The readers missed two of the 48 lesions $\geq 6 \mathrm{~mm}$ with PV and three of the 48 with SV, all related to perceptive or interpretation errors.

The high per-polyp sensitivity and the high specificity in our study can be explained by the following facts. As described previously, an effective full cathartic bowel preparation and tagging protocol, as well as automatic $\mathrm{CO}_{2}$ insufflation, were used [11]. Imaging was performed on 64-slice CT with isotropic $0.4-\mathrm{mm}$ spatial resolution, enabling high-quality $3 \mathrm{D}$ reconstructed images. In addition, both readers were experts in CTC, using primary 3D reading in their daily routine work, and were familiar with the reading PV software. There is an emerging consensus that one of the most important factors that affects the diagnostic performance of CTC is the dedicated training and experience of the radiologist who interprets the examination [28-30].

Compared to the 3D standard view, the potential limitations of the 3D panoramic view are luminal distortion and the short display time for blind spots and for the back view. Increasing surface visibility by flattening a 3D structure into a $2 \mathrm{D}$ image, or by changing viewing angles, is likely to suffer from the fact that the luminal anatomy is distorted, especially in areas of flexures or in suboptimally distended segments.

We recognised a higher degree of lesion distortions closer to the display periphery and compared with the "frontal view" with no deformation. Potential blind spots and the back view will consequently be displayed only outside the "frontal view," and lesions located in such areas of the mucosa will be distorted, which may reduce conspicuity compared with an undistorted lesion seen 
Fig. 4 a-d Sessile, 11-mm lobulated polyp located between two narrow standing, haustral folds in a 58-year-old asymptomatic male patient. Note that tagged material is colour labelled in $3 \mathrm{D}$ views. a The polyp is not visible either with the retrograde or with the antegrade (not shown) standard view. b Only the orthogonal standard virtual endoscopic perspective as well as $\mathbf{c}$ the unidirectional retrograde panoramic view display the lesion between the folds (arrows). Note the luminal distortion on the display periphery. d Transverse 2D image shows the lesion located between the folds (arrow)
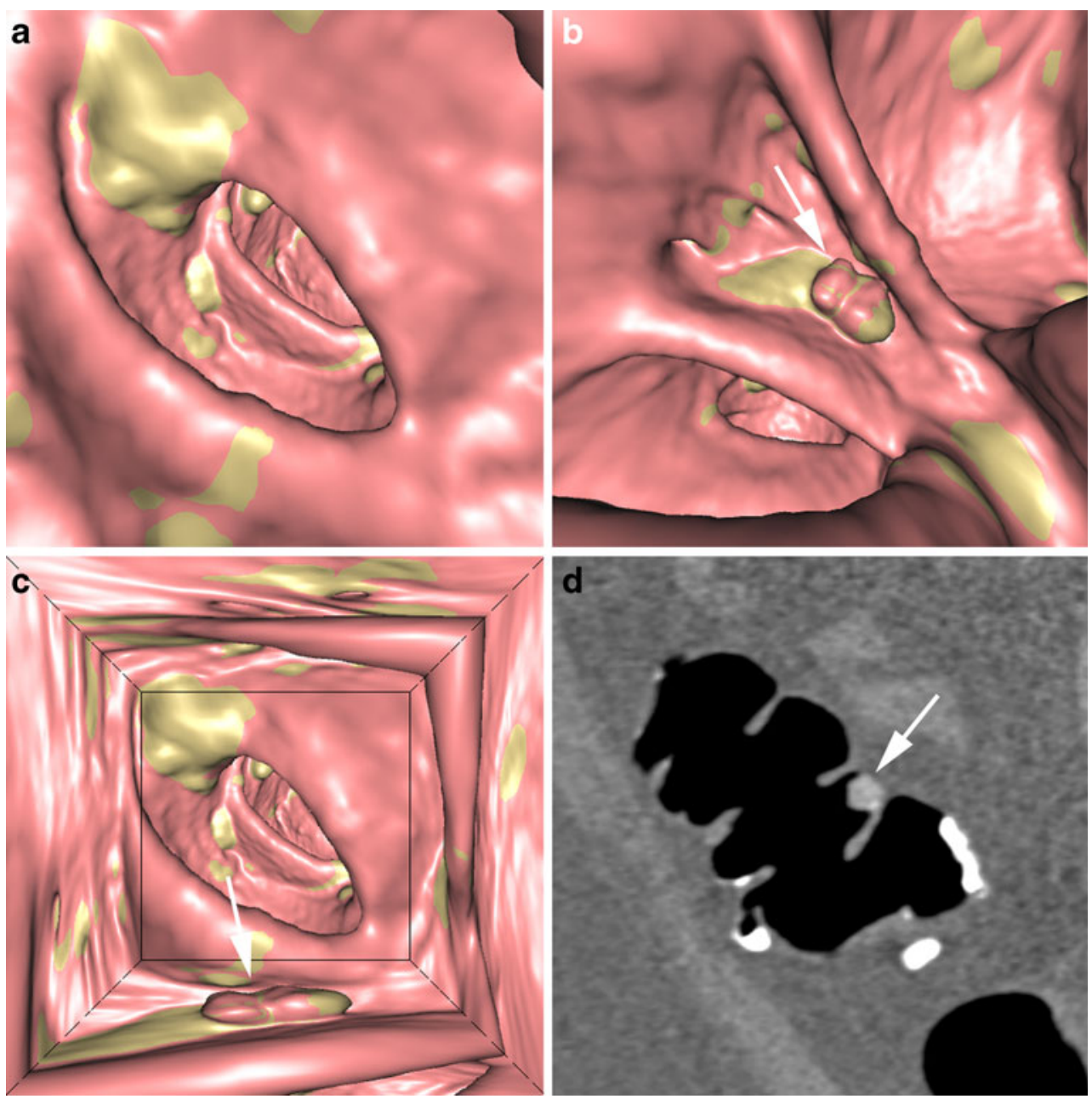

en face on the frontal view. Lesions located in blind spots or only visible on the back view will only be displayed for a short period of time when appearing only on the display periphery on the side and back projections, resulting in a reduced "polyp display time."

Although neither factor affected the readers' performance in the present evaluation, it might be possible that a less experienced reader who was unfamiliar with the software would perform differently. Therefore, because of the short lesion display time of some lesions and the increased luminal distortion, the 3D panoramic view requires dedicated reader expertise.

A recent study by Lenhart et al. performed with a different display version of the algorithm, by using a circular rather than a square display, demonstrated in a smaller, partially symptomatic population significantly higher PV reading times than the present study [31]. However, as stated in the manuscript, this might be related to the minimal prior experience of the readers with the panoramic view algorithm.

It is most likely that each different intraluminal view has a learning curve, and dedicated training may improve perception, especially in blind spots. Therefore, further studies are needed to evaluate the feasibility of such tools.

Our study has some limitations. It should be mentioned that calculations of the percentage of surface coverage do not include the mucosal endoluminal surface of collapsed colonic segments and may be affected by the presence of residual fluid. As both 3D endoluminal visualisation approaches are thereby hampered in the same way, the relative difference between the two visualisations should not be compromised by this circumstance.

Because the readers reviewed each patient dataset twice, one could argue that there might have been a learning bias between the two readings. We chose a time interval of 6-15 months between the two reading sessions, and patients were evaluated in a random fashion. In addition, both 3D display designs are relatively different, as described above, so that potential similarities in the display of morphology or intraluminal location, potentially biasing the reader, were minimised.

The apparently high prevalence of polyps is related to the fact that polyps $<6 \mathrm{~mm}$, as well as hyperplastic and 
adenomatous polyps, were included. When focusing on adenomas $\geq 6 \mathrm{~mm}$ or advanced lesions $>10 \mathrm{~mm}$ with a villous histology, the prevalence is in very good agreement with the prevalence of lesions in the asymptomatic German population, which has, in the meantime, been documented for more than 2.2 million persons [32].

In summary, we conclude that 3D unidirectional retrograde data evaluation with a $3 \mathrm{D}$ panoramic view significantly reduces the reading time with no significant differences in sensitivity compared with bidirectional 3D standard view. This technique visualises the complete colonic mucosa in a unidirectional fly-through, whereas standard 3D tools require bidirectional fly-through to achieve complete visualisation.

\section{References}

1. Taylor SA, Laghi A, Lefere P, Halligan S, Stoker J (2007) European Society of Gastrointestinal and Abdominal Radiology (ESGAR): consensus statement on CT colonography. Eur Radiol 17:575-579

2. Johnson CD, Chen MH, Toledano AY et al (2008) Accuracy of CT colonography for detection of large adenomas and cancers. $\mathrm{N}$ Engl J Med 359:1207-1217

3. Regge D, Laudi C, Galatola G et al (2009) Diagnostic accuracy of computed tomographic colonography for the detection of advanced neoplasia in individuals at increased risk of colorectal cancer. Jama 301:2453-2461

4. Yasumoto T, Murakami T, Yamamoto H et al (2006) Assessment of two 3D MDCT colonography protocols for observation of colorectal polyps. AJR Am J Roentgenol 186:85-89

5. Fletcher JG, Johnson CD, Reed JE, Garry J (2001) Feasibility of planar virtual pathology: a new paradigm in volume-rendered CT colonography. J Comput Assist Tomogr 25:864-869

6. Vos FM, van Gelder RE, Serlie IW et al (2003) Three-dimensional display modes for CT colonography: conventional $3 \mathrm{D}$ virtual colonoscopy versus unfolded cube projection. Radiology 228:878-885

7. Kim SH, Lee JM, Eun HW et al (2007) Two- versus threedimensional colon evaluation with recently developed virtual dissection software for CT colonography. Radiology 244:852864

8. Pickhardt PJ, Taylor AJ, Gopal DV (2006) Surface visualization at 3D endoluminal CT colonography: degree of coverage and implications for polyp detection. Gastroenterology 130:1582-1587

9. Geiger B, Chefd'hotel C, Sudarsky S (2005) Panoramic views for virtual endoscopy. Med Image Comput Comput Assist Interv Int Conf Med Image Comput Comput Assist Interv 8(Pt 1):662-669

10. Christensen E (2007) Methodology of superiority vs. equivalence trials and non-inferiority trials. J Hepatol 46:947-954

11. Graser A, Stieber P, Nagel D et al (2009) Comparison of CT colonography, colonoscopy, sigmoidoscopy and faecal occult blood tests for the detection of advanced adenoma in an average risk population. Gut 58:241-248

12. Graser A, Wintersperger BJ, Suess C, Reiser MF, Becker CR (2006) Dose reduction and image quality in MDCT colonography using tube current modulation. AJR Am J Roentgenol 187:695-701

13. Pickhardt PJ, Choi JR, Hwang I et al (2003) Computed tomographic virtual colonoscopy to screen for colorectal neoplasia in asymptomatic adults. N Engl J Med 349:2191-2200
14. Zalis ME, Barish MA, Choi JR et al (2005) CT colonography reporting and data system: a consensus proposal. Radiology 236:3-9

15. Macari M, Bini EJ, Jacobs SL, Lange N, Lui YW (2003) Filling defects at CT colonography: pseudo- and diminutive lesions (the good), polyps (the bad), flat lesions, masses, and carcinomas (the ugly). Radiographics 23:1073-1091

16. Pickhardt PJ, Lee AD, Taylor AJ et al (2007) Primary 2D versus primary $3 \mathrm{D}$ polyp detection at screening $\mathrm{CT}$ colonography. AJR Am J Roentgenol 189:1451-1456

17. Neri E, Vannozzi F, Vagli P, Bardine A, Bartolozzi C (2006) Time efficiency of CT colonography: 2D vs 3D visualization. Comput Med Imaging Graph 30:175-180

18. Pickhardt PJ (2007) Screening CT colonography: how I do it. AJR Am J Roentgenol 189:290-298

19. Silva AC, Wellnitz CV, Hara AK (2006) Three-dimensional virtual dissection at CT colonography: unraveling the colon to search for lesions. Radiographics 26:1669-1686

20. East JE, Saunders BP, Boone D, Burling D, Halligan S, Taylor SA (2008) Uni- and bidirectional wide angle CT colonography: effect on missed areas, surface visualization, viewing time and polyp conspicuity. Eur Radiol 18:1910-1917

21. East JE, Saunders BP, Burling D, Boone D, Halligan S, Taylor SA (2007) Surface visualization at CT colonography simulated colonoscopy: effect of varying field of view and retrograde view. Am J Gastroenterol 102:2529-2535

22. Pickhardt PJ, Schumacher C, Kim DH (2009) Polyp detection at 3-dimensional endoluminal computed tomography colonography: sensitivity of one-way fly-through at 120 degrees field-of-view angle. J Comput Assist Tomogr 33:631-635

23. Lee SS, Park SH, Kim JK et al (2009) Panoramic endoluminal display with minimal image distortion using circumferential radial ray-casting for primary three-dimensional interpretation of $\mathrm{CT}$ colonography. Eur Radiol 19:1951-1959

24. Hoppe H, Quattropani C, Spreng A, Mattich J, Netzer P, Dinkel HP (2004) Virtual colon dissection with CT colonography compared with axial interpretation and conventional colonoscopy: preliminary results. AJR Am J Roentgenol 182:1151-1158

25. Juchems MS, Fleiter TR, Pauls S, Schmidt SA, Brambs HJ, Aschoff AJ (2006) CT colonography: comparison of a colon dissection display versus 3D endoluminal view for the detection of polyps. Eur Radiol 16:68-72

26. Pickhardt PJ (2005) CT colonography (virtual colonoscopy) for primary colorectal screening: challenges facing clinical implementation. Abdom Imaging 30:1-4

27. Mang T, Peloschek P, Plank C et al (2007) Effect of computeraided detection as a second reader in multidetector-row CT colonography. Eur Radiol 17:2598-2607

28. Soto JA, Barish MA, Yee J (2005) Reader training in CT colonography: how much is enough? Radiology 237:26-27

29. van Dam J, Cotton P, Johnson CD et al (2004) AGA future trends report: CT colonography. Gastroenterology 127:970-984

30. Taylor SA, Halligan S, Burling D et al (2004) CT colonography: effect of experience and training on reader performance. Eur Radiol 14:1025-1033

31. Lenhart DK, Babb J, Bonavita J, et al (2010) Comparison of a unidirectional panoramic 3D endoluminal interpretation technique to traditional 2D and bidirectional 3D interpretation techniques at CT colonography: preliminary observations. Clin Radiol 65:118125

32. Brenner H, Hoffmeister M, Brenner G, Altenhofen L, Haug U (2009) Expected reduction of colorectal cancer incidence within 8 years after introduction of the German screening colonoscopy programme: estimates based on $1,875,708$ screening colonoscopies. Eur J Cancer 45:2027-2033 